\title{
Hydrogels for Biomedical Applications
}

\author{
Ahmed Sabr \\ University of Bridgeport, Biomedical Engineering Department, Bridgeport, CT USA
}

\begin{abstract}
In this paper, a facile way has been proposed to prepare transparent, tough and flexible polyacrylamide (PAM) hydrogels which is composed of a dually crosslinked single network by chemical crosslinking of $\mathrm{N}, \mathrm{N}^{\prime}$ methylenebisacrylamide (BIS) and physical crosslinking of hydrophilic hexagonal boron nitride (h-BN) nanosheets. invasion of VSMC into MMP-14 selective peptide crosslinked hydrogels was diametrically opposite in nature to that of fibroblasts whereby VSMC showed a two-fold increase into these hydrogels relative to that observed in permissive hydrogels whilst fibroblasts had a relative two-fold decrease
\end{abstract}

Keywords: Hydrogels, Applications, polymeric, configuration

\section{INTRODUCTION}

Hydrogel products constitute a group of polymeric materials, the hydrophilic structure of which renders them capable of holding large amounts of water in their threedimensional networks. Extensive employment of these products in a number of industrial and environmental areas of application is considered to be of prime importance. As expected, natural hydrogels were gradually replaced by synthetic types due to their higher water absorption capacity, long service life, and wide varieties of raw chemical resources. Literature on this subject was found to be expanding, especially in the scientific areas of research. However, a number of publications and technical reports dealing with hydrogel products from the engineering points of view were examined to overview technological aspects covering this growing multidisciplinary field of research. The primary objective of this article is to review the literature concerning classification of hydrogels on different bases, physical and chemical characteristics of these products, and technical feasibility of their utilization. It also involved technologies adopted for hydrogel production together with process design implications, block diagrams, and optimized conditions of the preparation process. An innovated category of recent generations of hydrogel materials was also presented in some details. The materials of interest in this brief review are primarily hydrogels, which are polymer networks extensively swollen with water. Hydrophilic gels that are usually referred to as hydrogels are networks of polymer chains that are sometimes found as colloidal gels in which water is the dispersion medium [1].

Researchers, over the years, have defined hydrogels in many different ways. The most common of these is that hydrogel is a water-swollen, and cross-linked polymeric network produced by the simple reaction of one or more monomers. Another definition is that it is a polymeric material that exhibits the ability to swell and retain a significant fraction of water within its structure, but will not dissolve in water. Hydrogels have received considerable attention in the past 50 years, due to their exceptional promise in wide range of applications, and.
They possess also a degree of flexibility very similar to natural tissue due to their large water content.

The ability of hydrogels to absorb water arises from hydrophilic functional groups attached to the polymeric backbone, while their resistance to dissolution arises from cross-links between network chains. Many materials, both naturally occurring and synthetic, fit the definition of hydrogels.

During last two decades, natural Hydrogels were gradually replaced by synthetic hydrogels which has long service life, high capacity of water absorption, and high gel strength. Fortunately, synthetic polymers usually have well-defined structures that can be modified to yield tailor able degradability and functionality. Hydrogels can be synthesized from purely synthetic components. Also, it is stable in the conditions of sharp and strong fluctuations of temperatures.

Recently, hydrogels have been defined as two- or multicomponent systems consisting of a three-dimensional network of polymer chains and water that fills the space between macromolecules. Depending on the properties of the polymer (polymers) used, as well as on the nature and density of the network joints, such structures in an equilibrium can contain various amounts of water; typically in the swollen state, the mass fraction of water in a hydrogel is much higher than the mass fraction of polymer. In practice, to achieve high degrees of swelling, it is common to use synthetic polymers that are watersoluble when in non-cross-linked form [2].

Hydrogels may be synthesized in a number of "classical" chemical ways. These include one-step procedures like polymerization and parallel cross-linking of multifunctional monomers, as well as multiple step procedures involving synthesis of polymer molecules having reactive groups and their subsequent cross-linking, possibly also by reacting polymers with suitable crosslinking agents. The polymer engineer can design and 
synthesize polymer networks with molecular-scale control over structure such as cross-linking density and with tailored properties, such as biodegradation, mechanical strength, and chemical and biological response to stimuli.

\section{CLASSIFICATION ACCORDING TO POLYMERIC COMPOSITION}

The method of preparation leads to formations of some important classes of hydrogels. These can be exemplified by the following:

(a)Homopolymeric hydrogels are referred to polymer network derived from a single species of monomer, which is a basic structural unit comprising of any polymer network. Homopolymers may have cross-linked skeletal structure depending on the nature of the monomer and polymerization technique.

(b)Copolymeric hydrogels are comprised of two or more different monomer species with at least one hydrophilic component, arranged in a random, block or alternating configuration along the chain of the polymer network.

(c)Multipolymer Interpenetrating polymeric hydrogel (IPN), an important class of hydrogels, is made of two independent cross-linked synthetic and/or natural polymer component, contained in a network form. In semi-IPN hydrogel, one component is a cross-linked polymer and other component is a non-cross-linked polymer.

Extracellular mimetic hydrogels formed from peptide crosslinkers and polyethylene glycol monomers permit cell-controlled invasion. The use of matrix metalloproteinase specific peptides might further allow for selective control of different cell-type invasion. In this study, the invasion of fibroblasts and vascular smooth muscle cells (VSMC) into hydrogels polymerised with either a peptide generally permissive for matrix metalloproteinase (MMP) degradation or peptides preferentially cleaved by MMP-14 or MMP-9 enzymes were compared. The two cell-types invaded the MMP permissive hydrogel equally. However, invasion of VSMC into MMP-14 selective peptide crosslinked hydrogels was diametrically opposite in nature to that of fibroblasts whereby VSMC showed a two-fold increase into these hydrogels relative to that observed in permissive hydrogels whilst fibroblasts had a relative two-fold decrease ( $\mathrm{p}<$ 0.01 ). These findings are suggestive that invasion and growth of different cell-types in engineered synthetic extracellular matrix mimics may be controlled selectively by the choice of protease specific peptide crosslinker and this could have general utility in tissue regenerative and engineering approaches.

\section{CLASSIFICATION BASED ON CONFIGURATION}

The classification of hydrogels depends on their physical structure and chemical composition can be classified as follows:
(a)Amorphous (non-crystalline).

(b)Semicrystalline: A complex mixture of amorphous and crystalline phases.

(c)Crystalline.

Generally, hydrogels prepared by bulk polymerization have inherent weak structure. To improve the mechanical properties of a hydrogel, it can be grafted on surface coated onto a stronger support. This technique that involves the generation of free radicals onto a stronger support surface and then polymerizing monomers directly onto it as a result a chain of monomers are covalently bonded to the support. A variety of polymeric supports have been used for the synthesis of hydrogel by grafting techniques [3]. The functional features of an ideal hydrogel material can be listed as following :

-The highest absorption capacity (maximum equilibrium swelling) in saline.

-Desired rate of absorption (preferred particle size and porosity) depending on the application requirement.

-The highest absorbency under load (AUL).

- The lowest soluble content and residual monomer.

-The lowest price.

-The highest durability and stability in the swelling environment and during the storage.

-The highest biodegradability without formation of toxic species following the degradation.

•pH-neutrality after swelling in water.

-Colorlessness, odorlessness, and absolute non-toxic.

-Photo stability.

-Re-wetting capability (if required) the hydrogel has to be able to give back the imbibed solution or to maintain it; depending on the application requirement (e.g., in agricultural or hygienic applications).

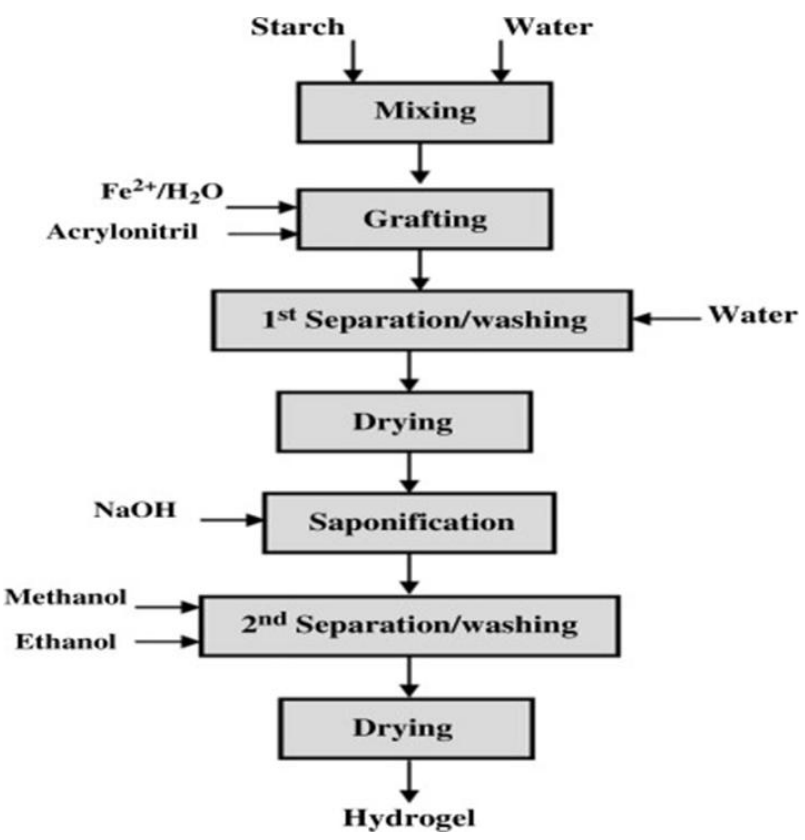


- Scaffolds in tissue engineering. When used as scaffolds, hydrogels may contain human cells to repair tissue. They mimic 3D microenvironment of cells.

- Hydrogel-coated wells have been used for cell culture.

- Environmentally sensitive hydrogels (also known as 'Smart Gels' or 'Intelligent Gels'). These hydrogels have the ability to sense changes of $\mathrm{pH}$, temperature, or the concentration of metabolite and release their load as result of such a change.

- Sustained-release drug delivery systems

- Providing absorption, desloughing and debriding of necrotic and fibrotic tissue

- Hydrogels that are responsive to specific molecules, such as glucose or antigens, can be used as biosensors, as well as in DDS.

- Disposable diapers where they absorb urine, or in sanitary napkins

- Contact lenses (silicone hydrogels, polyacrylamides, polymacon)

- EEG and ECG medical electrodes using hydrogels composed of cross-linked polymers (polyethylene oxide, polyAMPS and polyvinylpyrrolidone)

- Water gel explosives

- Rectal drug delivery and diagnosis

- Encapsulation of quantum dots.

\section{NANOCOMPOSITE HYDROGELS}

Nanocomposite hydrogels are also known as hybrid hydrogels, can be defined as highly hydrated polymeric networks, either physically or covalently crosslinked with each other and/or with nanoparticles or nanostructures. Nanocomposite hydrogels can mimic native tissue properties, structure and microenvironment due to their hydrated and interconnected porous structure. A wide range of nanoparticles, such as carbon-based, polymeric, ceramic, and metallic nanomaterials can be incorporated within the hydrogel structure to obtain nanocomposites with tailored functionality. Nanocomposite hydrogels can be engineered to possess superior physical, chemical, electrical, and biological properties. Many gels display thixotropy - they become fluid when agitated, but resolidify when resting. In general, gels are apparently solid, jelly-like materials. By replacing the liquid with gas it is possible to prepare aerogels, materials with exceptional properties including very low density, high specific surface areas, and excellent thermal insulation properties.

Hydrogel is a typical soft material that has a unique, threedimensionally cross-linked network structure swollen with a large amount of water. Basically, cross-linkings may be divided into two different types, i.e., chemical (covalent bonds) and physical (ionic bonds, hydrogen bonds, etc.). In the past decades, hydrogels have received increasing attention owing to a wide range of potential applications, including tissue engineering, drug delivery, membrane separation, electrolytes and soft robot. However, most of the existing natural and synthetic hydrogels have poor mechanical properties, which unfortunately limit their applications in many fields where tough and flexible hydrogels are necessary. In this sense, a surge of research has been reported on the development of nanocomposite hydrogels reinforced with various nanofillers, including clay nanosheets , silica nanoparticles, graphene oxide (GO) nanosheets and carbon nanotubes [4]. Recently, we have prepared a high-strength GO/PAA nanocomposite hydrogels composed of a single network with dual crosslinking points through dynamic ionic interactions. GO nanosheets are not only nanofiller with extraordinary mechanical strength and large surface area, but also serve as analogous crosslinking points in the gel network, which cooperate with reversible ionic cross-linking points among the polymer chains to endow the gel with super toughness and stretchability [5].

\section{CONCLUSION}

In conclusion, the direction of where smart hydrogels will go in the future should be addressed. Yes, all scientific evidence seems to indicate that basic and translational research in hydrogels has a bright future. Numerous new designs, e.g. involving protein domains containing noncanonical amino acids], successful attempts to control the morphology of self-assembling peptide fibers, artificial glycoproteins for controlling cell responses, hydrogels as the building material for microchemotaxis devices, enhanced use of DNA recognition motifs and improved synthetic methods demonstrate the versatility of the hybrid hydrogel approach. An outstanding example of the potential of stimuli-sensitive hydrogels in the development of bionanotechnology products is the design of optical systems that do not require mechanical components. Jiang's laboratory developed a tunable liquid lens that permits autonomous focusing. The design was based on a temperature-sensitive hydrogel integrated into a microfluidic system.

Obviously, there will be set-backs on the way forward but the scientific and translational potential of hydrogel biomaterials makes me confident in predicting a smart future.

\section{REFERENCES}

[1] Bracher, M., Bezuidenhout, D., Lutolf, M. P., Franz, T., Sun, M., Zilla, P., \& Davies, N. H. (2013). Cell specific ingrowth hydrogels. Biomaterials, 34(28), 6797-6803.

[2] Duan, Z.-Q., Zhong, M., Shi, F.-K., \& Xie, X.-M. Transparent hBN/polyacrylamide nanocomposite hydrogels with enhanced mechanical properties. Chinese Chemical Letters.

[3] Enas M, Ahmed, 2015 hydrogel: preparation, characterization and application

[4] Beebe, D. J., Moore, J. S., Bauer, J. M., Yu, Q., Liu, R. H., Devadoss, C., \& Jo, B. H. (2000). Functional hydrogel structures for autonomous flow control inside microfluidic channels. Nature, 404(6778), 588-590.

[5] Holtz, J. H., \& Asher, S. A. (1997). Polymerized colloidal crystal hydrogel films as intelligent chemical sensing materials. Nature, 389(6653), 829-832. 